\title{
Adaptação Cultural de Intervenções Preventivas em Saúde Mental Baseadas em Evidências
}

\author{
Jordana Calil Lopes de Menezest ${ }^{1}$ \\ Sheila Giardini Murta \\ ${ }^{1}$ Universidade de Brasilia, Brasilia, DF
}

\begin{abstract}
Resumo
A adaptação cultural de intervenções preventivas em saúde mental baseada em evidências tem sido largamente praticada ao redor do mundo. No Brasil, estudos com esse foco são raros. Tal processo deve ser sistemático para alcançar, por um lado, a preservação dos componentes centrais da intervenção original e, por outro, a sensibilidade cultural para com a cultura adotante de modo a respeitar a sua identidade, valores e necessidades. Realizou-se uma revisão narrativa acerca de modelos de adaptação cultural de intervenções preventivas importadas - Planned Intervention Adaptation (PLA) Protocol; Strengthening Families Program Adaptation Model (SFP_Ad); e ADAPT-ITT - cujas etapas e características foram analisadas. Conclui-se que a adaptação cultural de programas preventivos em saúde mental deve basear-se em estudos empíricos, práticas colaborativas e sensibilidade ética. Estudos futuros devem investigar o custo-benefício do investimento na adaptação cultural de intervenções internacionais versus o desenvolvimento de intervenções locais, para os quais são necessárias capacidades diversas.

Palavras-chave: prevenção, adaptação cultural, prática baseada em evidências
\end{abstract}

\section{Cultural Adaptation of Evidence Based Prevention Interventions in Mental Health}

\begin{abstract}
The cultural adaptation of evidence-based mental health preventive interventions has been widely practiced around the world. In Brazil, studies with this focus are rare. Such process must be systematic in order to achieve, on the one hand, the preservation of the core components of the original intervention and, on the other hand, the cultural sensitivity towards the adopting culture to respect its identity, values and needs. A narrative review on models of cultural adaptation of imported preventive interventions was carried out, including the Planned Intervention Adaptation (PIA) Protocol; Strengthening Families Program Adaptation Model (SFP_Ad); and the ADAPT-ITT, whose steps and characteristics were analyzed. It is concluded that the cultural adaptation of preventive programs in mental health should be based on empirical studies, collaborative practices and ethical sensitivity. Future studies should investigate the cost-effectiveness of investing in the cultural adaptation of international interventions versus the development of local interventions, for which different capacities are required.

Keywords: Prevention; cultural adaptation; evidence-based practice
\end{abstract}

\section{Adaptación Cultural de Intervenciones Preventivas en Salud Mental Basadas en Evidencias}

\begin{abstract}
Resumen
La adaptación cultural de intervenciones preventivas en salud mental basada en evidencias, ha sido ampliamente practicada alrededor del mundo. En Brasil, son escasos los estudios con este enfoque. Este proceso debe ser sistemático para alcanzar, por un lado, la preservación de los componentes centrales de la intervención original y, por otro, la sensibilidad con la cultura adoptante para respetar su identidad, valores y necesidades. Se realizó una revisión narrativa acerca de modelos de adaptación cultural de intervenciones preventivas importadas - Planned Intervention Adaptation (PIA) Protocol; (SFP_Ad); y ADAPT-ITT - cuyas etapas y características se analizaron. Se concluye que la adaptación cultural de programas preventivos en salud mental debe basarse en estudios empíricos, prácticas colaborativas y sensibilidad ética. Estudios futuros deben investigar el costo-beneficio de la inversión, en la adaptación cultural de intervenciones internacionales, con relación al desarrollo de intervenciones locales, para las cuales son necesarias capacidades diversas.

Palabras-clave: prevención; adaptación cultural; práctica basada en evidencias
\end{abstract}

O abuso de drogas, violência, depressão são alguns dos vários problemas não exclusivos do Brasil e que demandam intervenções preventivas (Andreou, 2015; Bromet et al., 2011; Moll, Elias, Gomes, Silva, \& Santos, 2015; Observatorio Interamericano de Drogas, 2015; Ronzani \& Silveira, 2014; Ruotti, Alves, \& Cubas, 2006). Muitos pesquisadores brasileiros dedicam-se a estudar a prevenção desses e de outros problemas mentais, emocionais e comportamentais (Müller, Paul,
\& Santos, 2008; Salgado, Senra, \& Lourenço, 2014; Silveira, Silvares, \& Marton, 2003; Teixeira et al., 2014). No entanto, as intervenções preventivas produzidas no Brasil carecem de pesquisas para verificar evidências de eficácia e efetividade que as tornariam passíveis de difusão em grande escala (Abreu, Miranda \& Murta, 2016; Menezes \& Murta, 2015).

$\mathrm{Na}$ literatura internacional existem programas preventivos desenvolvidos com evidências de eficácia e 
efetividade que poderiam ser adaptados para o contexto brasileiro, poupando assim o tempo e os custos que envolvem a elaboração de uma intervenção. Coloca-se aqui a questão central deste trabalho: quando as melhores evidências de prevenção em saúde mental são de uma intervenção internacional, como pode ocorrer a adaptação cultural dessa intervenção para o Brasil? Quais premissas metodológicas podem ser utilizadas para essa adaptação?

O presente estudo tem como objetivo discutir a adaptação cultural de intervenções preventivas em saúde mental baseadas em evidências como uma possibilidade para a pesquisa em prevenção no Brasil. Para isso, serão abordados: (a) aspectos conceituais da adaptação cultural; (b) questões relacionadas à adaptação cultural e fidelidade; (c) modelos para a realização de adaptações culturais de intervenções; e (d) desafios na área e agenda de pesquisa.

\section{Adaptação Cultural e seus Aspectos Conceituais}

A adaptação cultural de intervenções preventivas em saúde mental é escassa e recente na produção científica brasileira (D’Tôlis, 2018; Menezes, 2017). Achados de uma revisão sistemática acerca da pesquisa em prevenção em saúde mental no Brasil revelaram que a maior parte dos estudos nacionais produzidos até janeiro de 2012 concentrava-se, em sua grande maioria, na análise de necessidades para atuação na atenção básica à saúde, avaliação de fatores de risco e proteção para desfechos diversos em saúde e estudos piloto de intervenções preventivas. A té o período analisado, foram identificados apenas sete estudos de avaliação de eficácia e efetividade e nenhum estudo de adaptação cultural de intervenções (Abreu \& Murta, 2016).

A adaptação cultural de uma intervenção baseada em evidências corresponde à sua modificação sistemática para ajustá-la à cultura, ao contexto e à linguagem do público-alvo, considerando os seus valores, significados e padrões culturais (Castro, Barrera, \& Steiker, 2010). Uma intervenção pode requerer adaptação cultural quando é produzida em um contexto e adotada em outro cuja cultura difere substancialmente daquela em que foi originalmente produzida. Essa modificação está associada ao conceito de sensibilidade cultural. Entende-se por sensibilidade cultural a intensidade com que as características culturais, como normas, valores, padrões de comportamento, crenças, aspectos ambientais, históricos e sociais estão incluídas na intervenção, em seus materiais, forma de entrega e proposta de avaliação (Resnicow, Soler, Braithwaite, Ahulwalia, \& Butler, 2000).

A sensibilidade cultural tem duas dimensões: a estrutura superficial e a estrutura profunda. A primeira se refere a alterações nos materiais e mensagens do programa considerando características sociais e comportamentais da população-alvo. Ela corresponde ao quanto as mensagens, materiais e formas de transmissão da intervenção estão adequados às características da população-alvo. Está relacionada ao engajamento, adesão e motivação dos participantes a intervenção, o que por sua vez influencia diretamente na efetividade do programa (Berkel, Mauricio, Schoenfelder, \& Sandler, 2011). As mudanças na estrutura superficial do programa também podem torná-lo mais compreensível e culturalmente relevante. A relevância cultural representa o quanto o conteúdo e materiais do programa são aplicáveis ao dia a dia dos participantes (Resnicow et al., 2000). Outros autores a definem como o quanto as intervenções são consistentes com os valores, as crenças e os resultados desejados por uma determinada comunidade (Kumpfer, Alvarado, Smith, \& Bellamy, 2002).

A estrutura profunda, por sua vez, está relacionada ao comportamento saudável que a intervenção busca promover e aos aspectos culturais, sociais, ambientais e psicológicos da população-alvo que estão associados a esse comportamento saudável. Dessa forma, envolve alterações no conteúdo do programa para torná-lo mais adequado à realidade dos participantes em termos de valores, crenças, normas e outros aspectos culturais característicos dos participantes. Esse tipo de adequação da intervenção configura-se como um compromisso ético do pesquisador com a comunidade na medida em que a torna responsiva às necessidades da população-alvo (Reese \& Vera, 2007).

Por outro lado, ajustes na estrutura profunda de uma intervenção podem ocasionar a modificação de seus elementos centrais, que são os mecanismos responsáveis por seus efeitos (Resnicow et al., 2000; Sandoval, Corrêa, \& Abreu, 2015). Por isso, há relutância de alguns pesquisadores na área da adaptação cultural em realizar alterações na estrutura profunda das intervenções (Falicov, 2009). Tal resistência decorre do fato de que nem sempre os limites entre a estrutura profunda e os componentes centrais da intervenção estão bem definidos. Essa distinção é feita por aqueles que desenvolveram a versão original do programa por meio de estudos de mediação. Todavia, estudos de mediação nem sempre são possíveis ou ocorrem sem que todos os componentes centrais da intervenção sejam incluídos. Desse 
modo, a modificação da estrutura profunda da intervenção necessita ser planejada de maneira cuidadosa para que os componentes centrais do programa e sua consequente efetividade não sejam afetados (Gottfredson et al., 2015).

Por meio da adaptação cultural, pretende-se que o programa transferido para um novo contexto cultural seja percebido pelos participantes como atrativo, motivador, claro, compreensível, pertinente e relevante (Castro et al., 2010). Isso pode ser alcançado se o processo de adaptação cultural for conduzido por equipes com competência cultural, isto é, conhecedoras da cultura local (Resnicow et al., 2000). Quando isso ocorre, as alterações na intervenção tendem a ser sensíveis culturamente e podem facilitar a sua implementação e sustentabilidade. Por outro lado, essas mudanças também podem comprometer a fidelidade da intervenção (Stirman, Miller, Toder, \& Calloway, 2013), se as adaptações realizadas desconsiderarem os mecanismos responsáveis por sua efetividade.

\section{Adaptação Cultural e Fidelidade}

Uma questão largamente debatida nas pesquisas na área da adaptação cultural de intervenções diz respeito ao dilema entre manter a fidelidade à intervenção original e adaptá-la às características da população-alvo. A preservação da fidelidade durante o processo de adaptação cultural requer que a implementação da intervenção mantenha os seus componentes centrais e atividades e processos ligados aos resultados esperados (Segrott et al., 2013).

Evidências de uma ampla revisão sistemática que examinou a influência da qualidade da implementação sobre os resultados de 542 programas preventivos e os fatores que afetaram a implementação apontaram que, em geral, coexistem esforços para manter a fidelidade e adaptar as intervenções às necessidades e características do público-alvo (Durlak \& DuPre, 2008). De acordo com tal revisão, a qualidade da implementação dos programas preventivos impacta seus resultados e sofre forte influência de fatores contextuais, tais como elementos da comunidade, da qualidade da supervisão e treinamento oferecidos e da capacidade organizacional para implementação. Esses fatores representam importantes demandas para adaptação dos programas e, nesse sentido, customizações à realidade local podem ser indispensáveis e servir ao aprimoramento da implementação.

A produção de intervenções culturalmente adaptadas e íntegras em seu conteúdo requer cuidados e estratégias específicas. Alguns autores argumentam sobre a importância de as adaptações serem realizadas de modo sistemático, com base em evidências e com os elementos centrais da intervenção preservados (Kumpfer et al., 2002). A adaptação sistematizada contribui para a manutenção da fidelidade da intervenção original. Por isso, pesquisadores da área da adaptação cultural elaboraram estratégias para possibilitar a adequação da intervenção conservando a fidelidade (Marsiglia \& Booth, 2015).

\section{Modelos para a Adaptação Cultural de Intervenções}

A adaptação cultural quando feita de modo sistemático e em parceria com a comunidade aumenta a probabilidade de que esta venha a aderir ao programa e que possa se sustentar ao longo do tempo (Castro, Barrera, \& Martinez, 2004), uma vez que a comunidade detém o conhecimento sobre seus valores, crenças e cultura, o que pode tornar a intervenção responsiva às suas necessidades. Isso aumenta a relevância cultural da intervenção, promove o engajamento da comunidade e influencia positivamente os resultados (Berkel et al., 2011; Kumpfer et al., 2002).

A adaptação cultural de uma intervenção com vistas a aumentar sua relevância cultural pode requerer ajustes em sua estrutura superficial e profunda. Para que não haja prejuízos em relação à efetividade, é necessário sistematizar o processo de implementação e adaptação da intervenção (Berkel et al., 2011). Ferrer-Wreder, Sundell e Mansoory (2012) realizaram uma revisão de nove modelos que se propõem a sistematizar o processo de adaptação cultural de intervenções baseadas em evidências. Considerando o propósito deste estudo que é discutir o processo de adaptação cultural de intervenções preventivas internacionais para o Brasil, realizou-se uma análise desses nove modelos para identificar quais deles se destinam a adaptar intervenções preventivas para outros países. Verificou que, entre os nove modelos revisados, somente três se destinam à adaptação cultural de programas preventivos para outros países, cinco tratam da adaptação de uma intervenção para subgrupos culturais da própria população a que foi destinada a intervenção e uma delas se enquadra em ambas as categorias. Os modelos identificados que se destinam a adaptação cultural de intervenções para outros países são: Planned Intervention Adaptation (PLA) Protocol; Strengthening families program adaptation model (SFP_Ad); e ADAPT-ITT (Ferrer-Wreder et al., 2012).

Considerando que a adaptação cultural de um programa dentro de um mesmo país e para fins não 
preventivos pode ter especificidades que escapam ao escopo da presente análise, neste artigo serão analisados os três modelos que atenderam aos critérios propostos: Planned Intervention Adaptation (PLA) Protocol; ADAPT-ITT; e Strengthening families program adaptation model (SFP-Ad).

\section{Planned Intervention Adaptation (PLA) Protocol}

O Protocolo para Adaptação Planejada de Intervenções (Sundell \& Ferrer-Wreder, 2014, p. 51; tradução nossa) é um modelo de adaptação de programas de intervenção baseada no conceito de sensibilidade cultural de Resnicow et al. (2000). Portanto, a intervenção escolhida deve apresentar clareza em sua estrutura superficial e profunda (Ferrer-Wreder et al., 2012).

O Protocolo para Adaptação Planejada de Intervenções tem como elemento central a pesquisa e, por isso, propõe a elaboração de duas versões da intervenção a ser adaptada que são comparadas e testadas empiricamente para a avaliação de sua eficácia. Destarte, é necessário que a equipe envolvida na adaptação inclua pesquisadores. O Protocolo é dividido em duas fases. (Ferrer-Wreder et al., 2012; Sundell \& Ferrer-Wreder, 2014).

Fase I. A Fase I é composta por cinco passos:

a) Seleção de uma intervenção com evidências de eficácia e efetividade seguida da composição de uma equipe com os desenvolvedores da intervenção original escolhida e uma equipe de interessados para adaptação e tradução dos materiais e instrumentos utilizados na intervenção. A equipe de adaptação deve recrutar uma amostra de participantes equivalentes àqueles que receberão a intervenção final e dividi-la em três subgrupos, dois para avaliar os instrumentos utilizados na intervenção original e o outro para avaliar os materiais e atividades da intervenção original.

b) O subgrupo 1 responderá aos instrumentos utilizados na intervenção original traduzidos e os instrumentos utilizados no novo contexto e os resultados servirão para verificar a equivalência cultural e a adequação dos instrumentos para a nova cultura.

c) O subgrupo 2 responderá aos instrumentos revisados na fase anterior e será submetido a um estudo transversal destinado a verificar se os componentes centrais da intervenção alcançam os resultados esperados com essa nova população. Dessa forma, o terceiro passo do Protocolo busca averiguar a possibilidade de generalização da estrutura profunda da intervenção para o novo contexto.

d) O subgrupo 3, após finalizado o estudo transversal, constitui um grupo focal para analisar a relevância cultural e a adequação dos materiais e atividades da intervenção, ou seja, da estrutura superficial do programa.

e) Com base nos estudos realizados nas etapas anteriores em relação à estrutura superficial e profunda do programa são elaboradas duas versões da intervenção, uma com alterações mínimas relacionadas à linguagem e a estrutura superficial do programa e outra com modificações profundas advindas das pesquisas empíricas realizadas nas fases anteriores. Ambas as versões são testadas em estudos piloto e os resultados desses estudos orientam novas mudanças nas duas versões do programa. Isso irá aprimorar as duas versões da intervenção que irão compor a Fase II do processo de adaptação cultural.

Fase II. Consiste em um estudo experimental com três condições: um grupo na condição de intervenção que receberá o programa com alterações em sua estrutura superficial, outro grupo também na condição de intervenção que receberá o programa com modificações profundas advindas das pesquisas empíricas realizadas nas fases anteriores e um terceiro grupo na condição controle recebendo uma intervenção comumente utilizada. Os resultados desse estudo de efetividade poderão indicar a necessidade de aprimoramento na intervenção levando a outro estudo experimental ou demonstrar a necessidade de difusão da versão da intervenção que apresentar mais evidências de efetividade. Os autores sugerem também a realização de estudos de mediação e moderação em relação aos resultados (Sundell \& Ferrer-Wreder, 2014).

O Protocolo para Adaptação Planejada de Intervenções destaca-se por ser uma forma de adaptação cultural conduzida por estudos empíricos com triangulação de métodos. Por conseguinte, envolve um rigor científico que produz dados mais confiáveis para guiar a escolha dos atores envolvidos no processo de adaptação sobre a efetividade da intervenção adaptada para a nova cultura. Isso é crucial para a tomada de decisão a respeito da difusão do programa. Além disso, ele envolve a análise da relevância cultural da intervenção, o que se relaciona à adesão, engajamento e à motivação da comunidade para com a intervenção.

Outra característica de destaque é a distinção entre uma intervenção com mudanças na estrutura superficial 
e outra intervenção com alterações na estrutura profunda. Isso previne a modificação de componentes centrais da intervenção, o que pode prejudicar a efetividade da mesma.

\section{ADAPT-ITT}

É um modelo para adaptação de intervenções criado inicialmente para a adaptação de intervenções preventivas ao HIV. Ele é dividido em oito fases (Wingood \& DiClemente, 2008).

Fase 1. Consiste na avaliação de necessidades com a população alvo por meio de grupos focais ou entrevistas.

Fase 2. É a fase de decisão a respeito da intervenção mais adequada às necessidades da população-alvo. Indica-se também que após escolher a intervenção se avalie os fatores de risco e proteção da população-alvo para verificar se a teoria da intervenção selecionada atende a esses fatores de risco e proteção.

Fase 3. É denominada fase de administração. Os autores sugerem a realização de um pré-teste conhecido como teste teatro que é utilizado pela área de marketing para testar produtos. Trata-se da apresentação da intervenção a um grupo de prováveis participantes e de pessoas interessadas que irão fornecer feedback sobre a adequação da intervenção à cultura local, através de questionários com perguntas abertas e fechadas e discussão em grupo.

Fase 4. Com base nas informações colhidas nas fases anteriores, ocorre a elaboração de uma versão da intervenção que mantenha a fidelidade aos componentes centrais e a teoria do programa. Também é desenvolvido um plano de adaptação que irá registrar o processo em detalhes.

Fase 5. Consiste na escolha de especialistas na área para ajudar no processo de adaptação cultural da intervenção.

Fase 6. É a chamada fase de integração. Nesse momento, a informação dada pelos especialistas é reunida aos resultados provenientes das fases anteriores para criar outra versão da intervenção adaptada. Essa versão 2 da intervenção tem seu conteúdo avaliado por meio de questionário. Os resultados dos questionários são analisados e uma nova versão da intervenção é criada, resultando daí a versão 3 .

Fase 7. Ocorre o treinamento das pessoas envolvidas na aplicação da intervenção.

Fase 8. Consiste em testar a versão 3 da intervenção adaptada. Primeiro, é conduzido um estudo piloto em pequena escala com cerca de 20 participantes, sem grupo de comparação e com o registro de comentários sobre a intervenção fornecidos pelos participantes. Este piloto da intervenção adaptada forncecerá dados para o ensaio randomizado controlado da intervenção adaptada com uma avaliação dos resultados, com avaliações de pré, pós-teste e follow-up após três meses, que ocorrerá em seguida.

Diferente do Protocolo para Adaptação Planejada de Intervenções, o modelo ADAPT-ITT prevê uma orientação para a escolha da intervenção iniciando o processo a partir da avaliação de necessidades e baseando a escolha da intervenção a ser adaptada nos fatores de risco e proteção da população-alvo. A avaliação de necessidades informa a quem se destinará a intervenção e os objetivos da intervenção. Isso é muito importante, pois maximiza as chances de que o problema ao qual a intervenção se destina de fato se configura como uma dificuldade da população. Nesta direção, a compatibilidade entre o programa oferecido e as necessidades do público-alvo pode favorecer a adesão à intervenção (Bartholomew, Parcel, Kok, Gottlieb, \& Fernández, 2011).

Outro ponto de destaque desse modelo consiste no desenvolvimento de um plano para a adaptação da intervenção, que consiste na descrição detalhada do procedimento de adaptação incluindo instrumentos de medida. Esse registro pormenorizado do processo de adaptação evita o comprometimento da fidelidade do programa (Durlak \& DuPre, 2008).

Embora ambos os modelos apresentados envolvam estudos randomizados, o ADAPT-ITT indica a realização de follow-up, o que é fundamental para verificar os efeitos da intervenção em longo prazo. O follow-up pode ajudar tanto na averiguação de manutenção dos resultados, quanto na investigação do possível surgimento de efeitos iatrogênicos decorrentes ou não da intervenção (Gottfredson et al., 2015).

\section{Strengthening families program adaptation model (SFP-Ad)}

O modelo de adaptação do Strengthening Families Program foi desenvolvido pelos autores do programa com o objetivo de orientar a disseminação internacional do programa (Kumpfer, Pinyuchon, Melo, \& Whiteside, 2008; Kumpfer, Xie, \& O’Driscoll, 2012). São onze passos a serem seguidos:

a) A avaliação de necessidades, que consiste no recolhimento de informações a respeito dos principais fatores de risco e proteção à saúde do público-alvo; 
b) Uma revisão de literatura visando selecionar o programa preventivo baseado em evidência mais adequado para diminuir os fatores de risco e aumentar os fatores de proteção específicos da população-alvo;

c) A criação de uma equipe de adaptação cultural que deve ser composta pelos desenvolvedores do programa original, a comunidade que receberá o programa e os profissionais que realizarão a tradução e adaptação;

d) A realização da tradução de todo o material do programa, inclusive com a adaptação de alguns trechos que já se mostram inadequados à cultura, mas com alterações mínimas;

e) A implementação preliminar com adaptação mínima para se obter informações a respeito das adaptações ainda necessárias;

f) A adaptação cultural com base na implementação preliminar, efetuando mudanças gradativas no material, atividades, linguagem típicas da estrutura superficial do programa;

g) A incorporação ao currículo dessas e de outras adaptações culturais necessárias com a aprovação dos desenvolvedores do programa;

h) A realização de um ou mais estudos com a aplicação do programa adaptado e avaliações pré e pósteste para verificar a eficácia da intervenção com as adaptações feitas;

i) A efetuação de ajustes para incluir ou eliminar novas adaptações culturais;

j) A difusão que consiste em implementar a versão culturalmente adaptada do programa a um número maior de pessoas que apresentem aspectos culturais similares e avaliar a efetividade dessas implementações;

k) A publicação do processo e resultados da adaptação do programa (Kumpfer et al., 2008).

Embora o modelo de adaptação cultural do Strengthening Families Program tenha sido desenvolvido para essa intervenção específica, a maneira como ele é descrito demonstra a possibilidade de sua generalização para o processo de adaptação de outros programas. Além das semelhanças citadas, esse modelo apresenta outros pontos em comum com os modelos discutidos previamente.
Os três modelos utilizam-se de estudos piloto ou preliminares. Esse tipo de estudo é relevante, pois constitui uma experiência prévia para verificar como o programa funcionaria na prática. Isso também fornece informações a respeito da viabilidade, compreensão e atratividade da intervenção (Bartholomew, Parcel, Kok, \& Gottlieb, 2011). Os três modelos também preveem a realização de estudos de eficácia e efetividade que contribuem para o rigor metodológico do processo de adaptação cultural.

Um diferencial do modelo do Strengthening Families Program é a inclusão dos desenvolvedores do programa na equipe de adaptação. Esse contato com os desenvolvedores do programa ajuda a prevenir alterações na intervenção que possam comprometer sua fidelidade ou seus componentes centrais. Outro destaque desse modelo é a previsão da difusão da intervenção com estudos de efetividade como uma etapa importante do processo de adaptação cultural. Deve-se salientar que a cada novo contexto de implementação da intervenção novas demandas culturais surgem, o que pode influenciar na efetividade da intervenção. Por consequência, é importante manter a avaliação da efetividade do programa a cada aplicação em novo contexto (Berkel et al., 2011). O incentivo à publicação do processo de adaptação e de seus resultados também é digno de nota nesse modelo, visto que favorece o desenvolvimento teórico da área e o aprimoramento do processo de adaptação cultural de intervenções (Kumpfer et al., 2008).

\section{Desafios e Agenda de Pesquisa}

$\mathrm{O}$ vasto desenvolvimento de pesquisas em prevenção em saúde mental no âmbito internacional tem colocado à disposição de pesquisadores uma gama de programas de prevenção em saúde mental com evidências de eficácia e efetividade que podem ser adaptados culturalmente para as diversas localidades. A importação e adaptação dessas intervenções tem ocorrido em vários países como Reino Unido, Irlanda, Itália, Polônia, Alemanha, Grécia, Holanda, Espanha (Orte-Socias \& Amer-Fernández, 2014), Suécia (OrteSocias \& Amer-Fernández, 2014; Skärstrand, Larsson, \& Andréasson, 2008), Honduras (Vasquez et al., 2010), Costa Rica, El Salvador, Peru (Ortega, Giannotta, Latina, \& Ciairano, 2012), Chile (Corea, Zubarew, Valenzuela, \& Salas, 2012), Bolívia, Colômbia e Equador (Orpinas et al., 2014).

A adoção de programas internacionais traz alguns desafios. O primeiro diz respeito ao custo elevado da compra das licenças e dos materiais das intervenções 
internacionais. Além do valor da intervenção original, devem ser previstos os gastos com a produção dos materiais traduzidos que, muitas vezes, incluem livros e vídeos. Comparativamente, o desenvolvimento de intervenções nacionais desde sua origem, customizadas à cultura brasileira, poderia ter custos mais baixos. Adicionalmente, pode-se especular que intervenções assim desenvolvidas poderiam ser vistas como tendo maior legitimidade e, por isto, maior aceitabilidade quando comparadas a intervenções adaptadas. Um incremento em políticas científicas que fomentem a produção nacional de intervenções preventivas, desde seu desenvolvimento à sua avaliação de eficácia e efetividade, poderia representar um importante impacto na disponibilização de tecnologias sociais aplicáveis à promoção de saúde e prevenção de agravos à saúde, assim como na formação profissional e na constituição de redes de pesquisa.

O segundo desafio refere-se ao fato de que não há garantia de que uma intervenção será efetiva em qualquer lugar, mesmo que ela o tenha sido em muitos países (Sundell, Ferrer-Wreder, \& Fraser, 2012). Por isso, torna-se crucial que a adaptação cultural seja feita de modo sistematizado, com cuidados metodológicos e orientada por um modelo (Kumpfer et al., 2008), para não incorrer no risco de desperdício de recursos. Um processo sistematizado de adaptação cultural pode ganhar ao incluir elementos descritos nos modelos ora analisados, tais como a realização de estudos empíricos, a formação de equipes culturalmente competentes e a ação colaborativa entre diversos atores interessados. É evidente, portanto, ser este um processo oneroso, que consome recursos humanos qualificados e econômicos, além de tempo.

Associado a isto, tem-se um terceiro desafio, que se refere à constituição da equipe envolvida na adaptação cultural da intervenção. Ela deve incluir pessoas competentes culturalmente, pesquisadores especialistas em métodos quantitativos e qualitativos, pessoal treinado para aplicar a intervenção, além de pessoas da própria comunidade que receberá o programa. Como foi descrito nos três modelos, a própria comunidade contribui para a adaptação da intervenção e é objetivo desse processo que a intervenção seja percebida pela população-alvo como pertinente, clara e atrativa (Castro et al., 2010). Neste sentido, a condução do processo de adaptação cultural à luz de pressupostos da pesquisa participativa baseada na comunidade é altamente recomendada, tendo como diretrizes a incorporação da sabedoria da comunidade, o compromisso de longo prazo entre as partes, o benefício mútuo e a coaprendizagem (Wallerstein \& Duran, 2006).

O quarto desafio é a questão ética que envolve a adaptação de uma intervenção. $O$ conflito entre os conteúdos da intervenção e valores da comunidade pode gerar preconceito cultural e favorecer práticas colonialistas. Isto prejudica a interpretação de comportamentos da comunidade que são vistos como fatores de risco ou de proteção para a prevenção de determinada condição. Como é sabido, a classificação de um comportamento como fator de risco ou de proteção é contextual e cultural (Eriksson, Cater, Andershed, \& Andershed, 2010). Além disso, o investimento em uma intervenção sem sua adequada adaptação cultural pode resultar no uso indevido de recursos do contexto adotante, com desperdício de dinheiro, tempo e prejuízos à comunidade que a receber. $\mathrm{Ou}$, ainda, pode resultar em intervenções insensíveis culturalmente, que desconsideram o contexto e a identidade locais, impondo aos novos usuários valores e roupagens alheias às suas. Portanto a sensibilidade ética deve guiar a sistematização do processo de adaptação cultural de intervenções, com vistas à não produção de malefícios, à geração de benefícios, à preservação da autonomia da comunidade e respeito à sua identidade.

Tanto o desenvolvimento quanto a adaptação cultural de intervenções são processos complexos que demandam investimento financeiro, planejamento, rigor científico, e equipes técnica, cultural e eticamente capacitadas. Estando estes recursos disponíveis, o investimento na adaptação cultural de uma intervenção preventiva em saúde mental com evidências de eficácia e efetividade pode contribuir para o desenvolvimento da área de prevenção em saúde mental no Brasil, bem como para a agilidade na oferta de serviços preventivos em saúde mental. Neste último aspecto, a principal vantagem seria a oferta de programas baseados em evidências de efetividade em um tempo mais curto do que o necessário para o desenvolvimento de intervenções e sua avaliação criteriosa ao longo de anos. Isto, contudo, não exime os diversos atores interessados da construção de recursos políticos e técnicos para a produção de conhecimento acerca do desenvolvimento e avaliação de intervenções nacionais neste campo.

Diversas questões de pesquisa podem ser listadas no que tange à adaptação cultural de intervenções para o Brasil. Sugere-se pesquisar a aplicabilidade desses modelos na adaptação cultural de programas para o Brasil. O custo-benefício do investimento na adaptação 
cultural de uma intervenção versus desenvolvimento de intervenções nacionais é outro alvo para pesquisa. Por fim, a validade social de intervenções adaptadas culturalmente deve ser também examinada. No conjunto, estudos guiados por modelos que examinem custos versus benefícios e a relevância social percebida de intervenções culturalmente adaptadas podem elucidar em que medida a adaptação cultural de intervenções produzidas internacionalmente constituem alternativa pertinente para a realidade brasileira.

\section{Referências}

Abreu, S., \& Murta, S. G. (2016). O estado da arte da pesquisa em prevenção em saúde mental no Brasil: uma revisão sistemática. Interação em Psicologia, 20, 101-111. doi: 10.5380/psi.v20i1.34790

Abreu, S., Miranda, A. A. V., \& Murta, S. G. (2016). Programas preventivos brasileiros: quem faz e como é feita a prevenção em saúde mental? Psico-USF, 21, 163-177. doi: 10.1590/1413-82712016210114

Andreou, E. (2015). School violence prevention: The youth development perspective. British Journal of Education, Society and Behavioural Science, 5(4), 389395. doi: 10.9734/BJESBS/2015/13265

Bartholomew, L. K., Parcel, G. S., Kok, G., Gottlieb N. H., \& Fernández, M. E. (2011). Planning health promotion programs: An intervention mapping approach. San Francisco: Jossey-Bass.

Berkel, C., Mauricio, A. M., Schoenfelder, E., \& Sandler, I. N. (2011). Putting the pieces together: An integrated model of program implementation. Prevention Science, 12(1), 23-33. doi: 10.1007/ s11121-010-0186-1

Bromet, E., Andrade, L. H., Hwang, I., Sampson, N. A., Alonso, J., De Girolamo, G., ... \& Karam, A. N. (2011). Cross-national epidemiology of DSM-IV major depressive episode. BMC Medicine, 9(1), 1. doi: 10.1186/1741-7015-9-90

Castro, F. G., Barrera, M., \& Martinez, C. R. (2004). The cultural adaptation of prevention interventions: Resolving tensions between fidelity and fit. Prevention Science, 5(1), 41-45. doi: 10.1023/B:PREV.0000013980.12412.cd

Castro, F. G., Barrera Jr. M., \& Steiker, L. K. H. (2010). Issues and challenges in the design of culturally adapated evidence-based interventions. Annual
Review of Clinical Psychology, 6, 213-239. doi: 10.1146/ annurev-clinpsy-033109-132032

Costa, L. F. (2011). Participação de famílias no Grupo Multifamiliar de adolescentes ofensores sexuais: vergonha e confiança. Psicologia clínica, 23(1), 185201. doi: 10.1590/S0103-56652011000100012

Corea M. L., Zubarew T., Valenzuela M. T., \& Salas F. (2012). Evaluación del programa "Familias fuertes: amor y límites" en familias con adolescentes de 10 a 14 años. Revista médica de Chile, 140(6), 726-731. doi: 10.4067/S0034-98872012000600005

D’Tôlis, P. O. A. O. (2018). Avaliação do processo de adaptação cultural de um programa preventivo em saúde mental (dissertação de mestrado não publicada). Universidade Federal de Santa Catarina, Santa Catarina.

Durlak, J. A., \& DuPre, E. P. (2008). Implementation matters: A review of research on the influence of implementation on program outcomes and the factors affecting implementation. American Journal of Community Psychology, 41(3-4), 327-350. doi: 10.1007/s10464-008-9165-0

Eriksson, I., Cater, A., Andershed, A., \& Andershed, H. (2010). What we know and need to know about factors that protect youth from problems: A review of previous reviews. Procedia Social and Behavioral Sciences, 5, 477-482. doi: 10.1016/j. sbspro.2010.07.127

Falicov, C. J. (2009). Commentary: On the wisdom and challenges of culturally attuned treatments for Latinos. Family Process, 48(2), 292-309. doi: 10.1111/j.1545-5300.2009.01282.x

Ferrer-Wreder, L., Sundell, K., \& Mansoory, S. (2012). Tinkering with perfection: Theory development in the intervention cultural adaptation field. Child and Youth Care Forum, 41, 149-171. doi: 10.1007/ s10566-011-9162-6

Gottfredson, D. C., Cook, T. D., Gardner, F. E. M., Gorman-Smith, D., Howe, G. W., Sandler, I. N., \& Zafft, K. M. (2015). Standards of evidence for efficacy, effectiveness, and scale-up research in prevention science: Next generation. Prevention Science, 16, 893-926. doi: 10.1007/s11121-015-0555-x

Kumpfer, K. L., Alvarado, R., Smith, P., Bellamy, N. (2002). Cultural sensitivity in universal familybased prevention interventions. Prevention Science, 3(3), 241-244. doi: 10.1023/A:1019902902119 
Kumpfer, K. L., Pinyuchon, M., Teixeriade de Melo, A., \& Whiteside, H. O. (2008). Cultural adaptation process for international dissemination of the Strengthening Families Program. Evaluation \& The Health Professions, 31, 226-239. doi: $10.1177 / 0163278708315926$

Kumpfer, K. L., Xie, J., \& O’Driscoll, R. (2012). Effectiveness of a culturally adapted Strengthening Families Program 12-16 Years for high risk Irish families. Child and Youth Care Forum. doi: 10.1007/ s10566-011-9168-0

Marsiglia, F. \& Booth, J. (2015). Cultural adaptation of interventions in real practice settings. Research on Social Work Practice, 25(4), 423-432. doi: 10.1177/1049731514535989

Menezes, J. C. L. (2017). Adaptação Cultural do Strengthening Families Program (10-14) UK para o Brasil (tese de doutorado não publicada). Universidade de Brasília, Brasília. Recuperado de http://repositorio.unb. $\mathrm{br} /$ handle/10482/24729

Menezes, J. C. L., \& Murta, S. G. (2015). Intervenções para o desenvolvimento positivo com crianças brasileiras: Uma revisão integrativa. Em Z. A. P. Del Prette, A. B. Soares, C. S. Pereira-Guizzo, M. F. Wagner \& V. B. R. Leme (Eds.), Habilidades sociais: Diálogos e intercâmbios sobre pesquisa e prática (pp. 273294). Novo Hamburgo: Sinopsys.

Ministério da Saúde. (2015). Versão brasileira adaptada do Strengthening Families Program - SFP 10-14 UK. Brasília, DF.

Moll, M. F., Elias, B. A. B., Gomes, B. F., Silva, L. D., \& Santos, L. F. R. D. (2015). Depressão infantil na ótica dos professores do ensino fundamental. Journal of Nursing and Health, 4(2), 135-42. Recuperado de https://periodicos.ufpel.edu.br/ojs2/index. $\mathrm{php} /$ enfermagem/article/view/4388/3914

Müller, A. C., Paul, C. L., \& Santos, N. I. S. (2008). Prevenção às drogas nas escolas: Uma experiência pensada a partir dos modelos de atenção em saúde. Estudos de Psicologia (Campinas), 25(4), 607-616. doi: 10.1590/S0103-166X2008000400015

Observatorio Interamericano de Drogas (OID). (2015). Informe del uso de drogas em las Américas (OAS Cataloging-in-Publication Data). Recuperado de http://www.cicad.oas.org/apps/Document. aspx?Id=3209

Psico-USF, Bragança Paulista, v. 23, n. 4, p. 681-691, out./dez. 2018
Orte-Socias, C., \& Amer-Fernández, J. (2014). Las adaptaciones culturales del Strengthening $\mathrm{Fa}$ milies Program en Europa. Un ejemplo de programa de educación familiar basado en evidencia. Estudios sobre Educación, 26, 175-195. doi: 10.15581/004.26.175-195

Orpinas, P., Ambrose, A., Maddaleno, M., Vulanovic, L., Mejia, M., Butrón, B., ... Soriano, I. (2014). Lessons learned in evaluating the Familias Fuertes program in three countries in Latin America. Revista Panamericana de Salud Pública, 36(6), 383-390. Recuperado de http://www.scielosp.org/pdf/ rpsp/v36n6/a05v36n6.pdf

Ortega, E., Giannotta, F., Latina, D., \& Ciairano, S. (2012). Cultural adaptation of the strengthening families program 10-14 to Italian families. Child \& Youth Care Forum, 41(2), 197-212. doi: 10.1007/ s10566-011-9170-6

Reese, L., \& Vera, E. M. (2007). Culturally relevant prevention the scientific and practical considerations of community-based programs. The Counseling Psychologist, 35(6), 763-778. doi: http://dx.doi. org/10.1177/0011000007304588

Resnicow, K., Soler, R., Braithwaite, R., Ahulwalia, J., \& Butler, J. (2000). Cultural sensitivity in substance use prevention. Journal of Community Psychology, 28, 271-290. doi: 10.1002/(SICI)15206629(200005)28:3<271::AID-JCOP4>3.0.CO;2-I

Ronzani, T. M., \& Silveira, P. S. (Orgs.). (2014). Prevenção ao uso de álcool e outras drogas no contexto escolar. Juíz de Fora: UFJF.

Ruotti, C., Alves, R., \& Cubas, V. O. (2006). Violência na escola: Um guia para pais e professores. São Paulo: Andhep.

Salgado, F. S., Senra, L. X., \& Lourenço, L. M. (2014). Effectiveness indicators of bullying intervention programs: A systematic review of the international literature. Estudos de Psicologia (Campinas), 31(2), 179-190. doi: 10.1590/0103-166X2014000200004

Sandoval, L. A. N., Côrrea, A. O., \& Abreu. S. (2015). Adaptação cultural de programas de prevenção e promoção em saúde mental baseados em evidências. Em S. G. Murta, C. Leandro-França \& L. Polejack (Eds.), Prevenção e promoção em saúde mental: Fundamentos, planejamento e estratégias de intervenção (pp. 249-262). Novo Hamburgo: Sinopsys. 
Segrott J., Holliday J., Rothwell H., Foxcroft, D., Murphy, S., Scourfield, J., Hood, K., \& Laurence, M. (2013). Cultural adaptation and intervention integrity: A response to Skarstrand, Sundell and Andreasson. European Journal of Public Health, 24, 354-55. doi: 10.1093/eurpub/cku039

Silveira, J. M., Silvares, E. F. M., \& Marton, S. A. (2003). Programas preventivos de comportamentos antissociais: Dificuldades na pesquisa e na implementação. Estudos de Psicologia (Campinas), 20(3), 59-67. doi: 10.1590/S0103-166X2003000300005

Skärstrand, E., Larsson, J., \& Andréasson, S. (2008). Cultural adaptation of the Strengthening Families Programme to a Swedish setting. Health Education, 108(4), 287-300. doi: 10.1108/09654280810884179

Stirman, S.W., Miller C.J., Toder, K., \& Calloway, A. (2013). Development of a framework and coding system for modifications and adaptations of evidence-based interventions. Implement Science, 8, 65. doi: 10.1186/1748-5908-8-65

Sundell, K., \& Ferrer-Wreder, L. (2014). The transportability of empirically supported interventions. Em A. Shlonsky \& R. Benbenishty (Eds.), From evidence to outcomes in child welfare: An international reader (pp. 41-58). New York, NY: Oxford University Press.

Sundell, K., Ferrer-Wreder, L., \& Fraser, M. W. (2014). Going Global a Model for Evaluating Empirically Supported Family-Based Interventions in New
Contexts. Evaluation \& The Health Professions, 37(2), 203-230. doi: 10.1177/0163278712469813

Teixeira, M. C. T. V., Seraceni, M. F. F., Suriano, R., Sant'ana, N. Z., Carreiro, L. R. R., \& Paula, C. S. D. (2014). Protective factors associated with emotional and behavioral problems in school children. Estudos de Psicologia (Campinas), 31(4), 539-548. doi: 10.1590/0103-166X2014000400008

Vasquez, M., Mesa, L., Almandarez, O., Santos, A., Matute, R., Diaz, L., . . . Saenz, K. (2010). Evaluation of a Strengthening Families (Familias Fuertes) Intervention for Parents and Adolescents in Honduras. The Southern Online Journal of Nursing Research, 10(3), 1-25. Recuperado de http://www.resourcenter.net/images/snrs/files/sojnr_articles2/ Vol10Num03Art01.pdf

Wallerstein N. B., \& Duran B. (2006). Using community-based participatory research to address healthdisparities. Health Promotion Practice, 7(3), 312323. doi/abs/10.1177/1524839906289376

Wingood, G. M., \& DiClemente, R. J. (2008). The ADAPT-ITT model: A model for adapting evidence based HIV interventions. Journal of Acquired Immunodeficiency Syndrome, 47(Suppl. 1), S40-S46. doi: 10.1097/QAI.0b013e3181605df1.

Recebido em: 20-02-2017

Reformulado em: 20-02-2018

Aprovado em: 04-04-2018 
Sobre as autoras:

Jordana Calil Lopes de Menezes é psicóloga pela PUC Goiás, especialista em Gestalt-terapia pelo ITGT, mestre e doutora em Psicologia Clínica e Cultura pela UnB, orientadora do ITGT, membro do GT-ANPEPP "Relações Interpessoais e Competência Social" e do Grupo de Estudos em Prevenção e Promoção de Saúde no Ciclo de Vida (GEPPSVida) e pesquisadora associada da UnB.

E-mail: jordanacalil@gmail.com

Sheila Giardini Murta é psicóloga (PUC Goiás), especialista em Análise Política e Políticas Públicas, mestre em Psicologia do Desenvolvimento Humano e doutora em Psicologia Social e do Trabalho (UnB), realizou estágio de doutoramento na Queensland University of Technology, pós-doutorado pela UFSCAR e Universidade de Maastricht - Holanda. É professora associada no Departamento de Psicologia Clínica do Instituto de Psicologia e orientadora no Programa de Pós-Graduação em Psicologia Clínica e Cultura (UnB), membro do GT-ANPEPP 'Relações Interpessoais e Competência Social” e coordenadora do GEPPSVida. E-mail: giardini@unb.br

Contato com as autoras:

Campus Universitário Darcy Ribeiro - ICC SUL, Instituto de Psicologia, Pós-Graduação do Departamento de Psicologia Social e do Trabalho - PST

Asa Norte

Brasília-DF, Brasil

CEP: 72910-000 